\title{
INEQUALITIES OF CHEBYSHEV-PÓLYA-SZEGÖ TYPE VIA GENERALIZED PROPORTIONAL FRACTIONAL INTEGRAL OPERATORS
}

\author{
SAAD IHSAN BUTT, AHMET OCAK AKDEMIR, ALPER EKINCI, \\ AND MUHAMMAD NADEEM
}

Received 27 May, 2020

\begin{abstract}
This study is an example of a solid connection between fractional analysis and inequality theory, and includes new inequalities of the Pólya-Szegö-Chebyshev type obtained with the help of Generalized Proportional Fractional integral operators. The results have been performed by using Generalized Proportional Fractional integral operators, some classical inequalities such as AM-GM inequality, Cauchy-Schwarz inequality and Taylor series expansion of exponential function. The findings give new approaches to some types of inequalities that have involving the product of two functions in inequality theory.
\end{abstract}

2010 Mathematics Subject Classification: 26A33; 26D10; 26D15

Keywords: Chebyshev inequality, Polya-Szegö type inequalities, GPF Polya-Szegö operator

\section{INTRODUCTION AND PRELIMINARIES}

Inequalities are a concept that contributes to the solution of many problems with their applications in different disciplines such as engineering, physics, statistics and economics as well as being used in many branches of mathematics. With the help of convex, differentiable, integrable, continuous, limited, synchronous functions or some other specially defined functions, many different types of inequality have been proved in the inequality theory field. These inequalities were brought to the literature under different names and later became functional with their applications. We will start by expressing Chebyshev inequality, one of the major inequality types of inequality theory (see [2]):

$$
T(f, g)=\frac{1}{b-a} \int_{a}^{b} f(x) g(x) d x-\left(\frac{1}{b-a} \int_{a}^{b} f(x) d x\right)\left(\frac{1}{b-a} \int_{a}^{b} g(x) d x\right)
$$

The research of the first author has been fully supported by H.E.C. Pakistan under NRPU project 7906. 
where $f$ and $g$ are two integrable functions which are synchronous on $[a, b]$, i.e.

$$
(f(x)-f(y))(g(x)-g(y)) \geq 0
$$

for any $x, y \in[a, b]$, then the Chebyshev inequality states that $T(f, g) \geq 0$.

Chebyshev inequality has been proven for synchronous functions, and has been the focus of researchers and many different versions have been obtained. We encourage interested readers to review the following articles. Chebyshev inequality has been proven for synchronous functions and has been the focus of researchers and many different versions have been obtained. We encourage interested readers to review the following articles [3, 14, 21] and [15].

Another interesting inequality is the Pólya-Szegö inequality, which gives boundaries for two functions that can be integrated and their product. This inequality is given as follows: (see [18])

$$
\frac{\int_{a}^{b} f^{2}(x) d x \int_{a}^{b} g^{2}(x) d x}{\left(\int_{a}^{b} f(x) g(x) d x\right)^{2}} \leq \frac{1}{4}\left(\sqrt{\frac{M N}{m n}}+\sqrt{\frac{m n}{M N}}\right)^{2}
$$

This inequality is very useful in proving Grüss and Chebyshev type inequalities. With the help of this inequality, a Chebyshev-Grüss type inequality is expressed by Dragomir and Diamond as follows in [7]:

Theorem 1. Let $f, g:[a, b] \rightarrow \mathbb{R}_{+}$be two integrable functions so that

$$
\begin{gathered}
0<m \leq f(x) \leq M<\infty \\
0<n \leq g(x) \leq N<\infty
\end{gathered}
$$

for $x \in[a, b]$. Then we have

$$
|T(f, g ; a, b)| \leq \frac{1}{4} \frac{(M-m)(N-n)}{\sqrt{m n M N}}\left(\frac{1}{b-a} \int_{a}^{b} f(x) d x\right)\left(\frac{1}{b-a} \int_{a}^{b} g(x) d x\right)
$$

The constant $\frac{1}{4}$ is best possible in (1.2) in the sense it can not be replaced by a smaller constant.

Remark 1 (see [7]). Assume that the inequality in (1.2) holds with a constant $c>0$, i.e.,

$$
|T(f, g ; a, b)| \leq c \frac{(M-m)(N-n)}{\sqrt{m n M N}}\left(\frac{1}{b-a} \int_{a}^{b} f(x) d x\right)\left(\frac{1}{b-a} \int_{a}^{b} g(x) d x\right)
$$

We choose the functions as $f=g$ with

$$
f(x)=\left\{\begin{aligned}
c m, & x \in\left[a, \frac{a+b}{2}\right] \\
M, & x \in\left[\frac{a+b}{2}, b\right]
\end{aligned}\right.
$$


where $0<m<M<\infty$, then

$$
m M \leq c(M-m)^{2}
$$

for any $0<m<M<\infty$. If in (1.3) we consider $m=1-\varepsilon, M=1+\varepsilon, \varepsilon \in(0,1)$, then we get $1-(\varepsilon)^{2} \leq 4 c$ for any $\varepsilon \in(0,1)$, which show that $c \geq \frac{1}{4}$.

Although fractional analysis origin dates back to the beginning of classical analysis, it has developed quite rapidly in recent years. Many mathematicians who researched in this field contributed to this development and made efforts to strengthen the relationship between fractional analysis and other fields. With the introduction of new fractional derivative and integral operators, the application opportunity for many real-world problems has been revealed. The majority of the new operators came to the fore with different features such as singularity, location and generalization, and gained functionality thanks to their effective use in application areas (see the papers $[1,4-6,8-10,12,13,16,17,19,20])$. Due to the intensive work on it, the RiemannLiouville integral operator is a prominent operator and is defined as follows.

Definition 1. Let $f \in L_{1}[a, b]$. The Riemann-Liouville integrals $J_{a+}^{\alpha} f$ and $J_{b-}^{\alpha} f$ of order $\alpha>0$ with $a \geq 0$ are defined by

$$
J_{a+}^{\alpha} f(t)=\frac{1}{\Gamma(\alpha)} \int_{a}^{t}(t-x)^{\alpha-1} f(x) d x, \quad t>a
$$

and

$$
J_{b-}^{\alpha} f(t)=\frac{1}{\Gamma(\alpha)} \int_{t}^{b}(x-t)^{\alpha-1} f(x) d x, \quad t<b
$$

respectively. Here $\Gamma(t)$ is the Gamma function and its definition is $\Gamma(t)=\int_{0}^{\infty} e^{-t} t^{x-1} d x$. It is to be noted that $J_{a+}^{0} f(t)=J_{b-}^{0} f(t)=f(t)$ in the case of $\alpha=1$, the fractional integral reduces to the classical integral.

We will continue with the Generalized Proportional Fractional integral operator, which has been described recently and has been the main source of motivation for many studies in the literature with its use in many areas, especially the inequality theory. In [11], Jarad et al. identified the proportional generalized fractional integrals that satisfy many important features as follows:

Definition 2. The left and right generalized proportional fracitonal integral operators are respectively defined by

$$
{ }_{a+} \mathfrak{J}^{\alpha, \lambda} f(t)=\frac{1}{\lambda^{\alpha} \Gamma(\alpha)} \int_{a}^{t} e^{\left[\frac{\lambda-1}{\lambda}(t-x)\right]}(t-x)^{\alpha-1} f(x) d x, \quad t>a
$$

and

$$
{ }_{b-\mathfrak{J}^{\alpha, \lambda}} f(t)=\frac{1}{\lambda^{\alpha} \Gamma(\alpha)} \int_{t}^{b} e^{\left[\frac{\lambda-1}{\lambda}(x-t)\right]}(x-t)^{\alpha-1} f(x) d x, \quad t<b
$$

where $\lambda \in(0,1]$ and $\alpha \in \mathbb{C}$ and $\mathbb{R}(\alpha)>0$. 
The main aim of this study is to obtain new Pólya-Szegö type inequalities by using Generalized Proportional Fractional integral operators. Taylor series expansion of exponential function is used in addition to some classical inequalities to obtain main results. The study is enriched by giving special cases of our results.

\section{MAIN RESULTS}

In this section, we prove certain Pólya-Szegö type integral inequalities for positive integral functions involving Generalized Proportional Fractional integral operator.

Lemma 1. Assume that $f$ and $g$ are two positive integrable function on $[0, \infty)$. If $v_{1}, v_{2}, w_{1}$ and $w_{2}$ are positive functions such that

$$
\begin{aligned}
& 0<v_{1}(\tau) \leq f(\tau) \leq v_{2}(\tau) \\
& 0<w_{1}(\tau) \leq g(\tau) \leq w_{2}(\tau)
\end{aligned}
$$

for $\tau \in[0, x], x>0$, then we have the following inequality;

$$
\frac{{ }_{0}^{G P F} I^{\alpha, p_{1}}\left[w_{1} w_{2} f^{2}\right](x)_{0}^{G P F} I^{\alpha, p_{1}}\left[v_{1} v_{2} g^{2}\right](x)}{\left({ }_{0}^{G P F} I^{\alpha, p_{1}}\left[\left(v_{1} w_{1}+v_{2} w_{2}\right) f g\right](x)\right)^{2}} \leq \frac{1}{4} .
$$

where $\alpha \in(n, n+1]$ and $n=1,2,3, \ldots$

Proof. From (2.1) for $\tau \in[0, x], x>0$, we can write

$$
\left(\frac{v_{2}(\tau)}{w_{1}(\tau)}-\frac{f(\tau)}{g(\tau)}\right) \geq 0
$$

and

$$
\left(\frac{f(\tau)}{g(\tau)}-\frac{v_{1}(\tau)}{w_{2}(\tau)}\right) \geq 0 .
$$

If we multiply (2.3) and (2.4) side by side, we have

$$
\left(\frac{v_{2}(\tau)}{w_{1}(\tau)}-\frac{f(\tau)}{g(\tau)}\right)\left(\frac{f(\tau)}{g(\tau)}-\frac{v_{1}(\tau)}{w_{2}(\tau)}\right) \geq 0
$$

This implies the following inequality,

$$
\left(v_{1}(\tau) w_{1}(\tau)+v_{2}(\tau) w_{2}(\tau)\right) f(\tau) g(\tau) \geq w_{1}(\tau) w_{2}(\tau) f^{2}(\tau)+v_{1}(\tau) v_{2}(\tau) g^{2}(\tau)
$$

Since all the functions are positive, $p_{1} \in(0,1], x \geq \tau$ and $x>0$, by multiplying both sides of (2.5) by $\frac{1}{p_{1}^{\alpha} \Gamma(\alpha)} e^{\frac{p_{1}-1}{p_{1}}(x-\tau)}(x-\tau)^{\alpha-1}$ and then integrating the resulting inequality with respect to $\tau$ over $(0, x)$, we get

$$
\frac{1}{p_{1}^{\alpha} \Gamma(x)} \int_{0}^{x} e^{\frac{p_{1}-1}{p_{1}}(x-\tau)}(x-\tau)^{\alpha-1}\left(v_{1}(\tau) w_{1}(\tau)+v_{2}(\tau) w_{2}(\tau)\right) f(\tau) g(\tau) d \tau
$$




$$
\begin{aligned}
\geq & \frac{1}{p_{1}^{\alpha} \Gamma(x)} \int_{0}^{x} e^{\frac{p_{1}-1}{p_{1}}(x-\tau)}(x-\tau)^{\alpha-1}\left(w_{1}(\tau) w_{2}(\tau) f^{2}(\tau)\right) d \tau \\
& +\frac{1}{p_{1}^{\alpha} \Gamma(x)} \int_{0}^{x} e^{\frac{p_{1}-1}{p_{1}}(x-\tau)}(x-\tau)^{\alpha-1} v_{1}(\tau) v_{2}(\tau) g^{2}(\tau) d \tau .
\end{aligned}
$$

Namely,

$$
{ }_{0}^{G P F} I^{\alpha, p_{1}}\left[\left(v_{1} w_{1}+v_{2} w_{2}\right) f g\right](x) \geq{ }_{0}^{G P F} I^{\alpha, p_{1}}\left[w_{1} w_{2} f^{2}\right](x)+{ }_{0}^{G P F} I^{\alpha, p_{1}}\left[v_{1} v_{2} g^{2}\right](x) \text {. }
$$

Applying the A.M-G.M inequality i.e $\left(a+b \geq 2 \sqrt{a b}, a, b \in \mathfrak{R}^{+}\right)$, we have

$$
{ }_{0}^{G P F} I^{\alpha, p_{1}}\left[\left(v_{1} w_{1}+v_{2} w_{2}\right) f g\right](x) \geq 2 \sqrt{{ }_{0}^{G P F} I^{\alpha, p_{1}}\left[w_{1} w_{2} f^{2}\right](x)_{0}^{G P F} I^{\alpha, p_{1}}\left[v_{1} v_{2} g^{2}\right](x)} .
$$

This can be written as

$$
{ }_{0}^{G P F} I^{\alpha, p_{1}}\left[w_{1} w_{2} f^{2}\right](x)_{0}^{G P F} I^{\alpha, p_{1}}\left[v_{1} v_{2} g^{2}\right](x) \leq \frac{1}{4}\left(\underset{0}{G P F} I^{\alpha, p_{1}}\left[\left(v_{1} w_{1}+v_{2} w_{2}\right) f g\right](x)\right)^{2}
$$

Corollary 1. If we take into account $v_{1}=m, v_{2}=m, w_{1}=n$ and $w_{2}=N$ in (2.2), then we have the following new inequality;

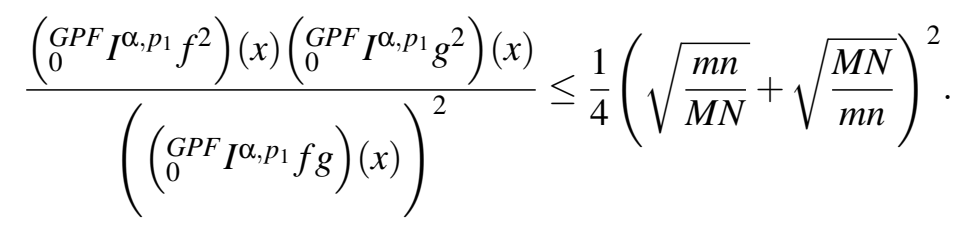

Lemma 2. Let $f$ and $g$ be two positive integrable functions on $[0, \infty)$. Assume that there exists four positive integrable functions $v_{1}, v_{2}, w_{1}$ and $w_{2}$ satisfying condition (2.1). Then the following inequality holds:

$$
\begin{aligned}
& { }_{0}^{G P F} I^{\alpha, p_{1}}\left[v_{1} v_{2}\right](x)_{0}^{G P F} I^{\beta, p_{2}}\left[w_{1} w_{2}\right](x) \times{ }_{0}^{G P F} I^{\alpha, p_{1}}\left[f^{2}\right](x)_{0}^{G P F} I^{\beta, p_{2}}\left[g^{2}\right](x) \\
\leq & \frac{1}{4}\left({ }_{0}^{G P F} I^{\alpha, p_{1}}\left[v_{1} f\right](x)_{0}^{G P F} I^{\beta, p_{2}}\left[w_{1} g\right](x)+{ }_{0}^{G P F} I^{\alpha, p_{1}}\left[v_{2} f\right](x)_{0}^{G P F} I^{\beta, p_{2}}\left[w_{2} g\right](x)\right)^{2}
\end{aligned}
$$

where $\alpha \in(n, n+1]$ and $\beta \in(k, k+1], n, k=0,1,2,3, \ldots$.

Proof. From (2.1), we get

$$
\left(\frac{v_{2}(\tau)}{w_{1}(\xi)}-\frac{f(\tau)}{g(\xi)}\right) \geq 0
$$

and

$$
\left(\frac{f(\tau)}{g(\xi)}-\frac{v_{1}(\tau)}{w_{2}(\xi)}\right) \geq 0
$$


Which leads to

$$
\left(\frac{v_{1}(\tau)}{w_{2}(\xi)}+\frac{v_{2}(\tau)}{w_{1}(\xi)}\right) \frac{f(\tau)}{g(\xi)} \geq \frac{f^{2}(\tau)}{g^{2}(\xi)}+\frac{v_{1}(\tau) v_{2}(\tau)}{w_{1}(\xi) w_{2}(\xi)} .
$$

Multiplying both sides of $(2.8)$ by $w_{1}(\xi) w_{2}(\xi) g^{2}(\xi)$, we have

$$
v_{1}(\tau) f(\tau) w_{1}(\xi) g(\xi)+v_{2}(\tau) f(\tau) w_{2}(\xi) g(\xi) \geq w_{1}(\xi) w_{2}(\xi) f^{2}(\tau)+v_{1}(\tau) v_{2}(\tau) g^{2}(\xi) \text {. }
$$

Multiplying both sides (2.9) by

$$
\frac{1}{p_{1}^{\alpha} \Gamma(\alpha)} \frac{1}{p_{2}^{\beta} \Gamma(\beta)} e^{\frac{p_{1}-1}{p_{1}}(x-\tau)}(x-\tau)^{\alpha-1} e^{\frac{p_{2}-1}{p_{2}}(x-\xi)}(x-\tau)^{\alpha-1}(x-\xi)^{\beta-1}
$$

and integrating the resulting inequality with respect to $\tau$ and $\xi$ over $(0, x)^{2}$, we get

$$
\begin{aligned}
& \frac{1}{p_{1}^{\alpha} \Gamma(\alpha)} \frac{1}{p_{2}^{\beta} \Gamma(\beta)} \\
& \int_{0}^{x} \int_{0}^{x} e^{\frac{p_{1}-1}{p_{1}}(x-\tau)} e^{\frac{p_{2}-1}{p_{2}}(x-\xi)}(x-\tau)^{\alpha-1}(x-\xi)^{\beta-1} v_{1}(\tau) f(\tau) w_{1}(\xi) g(\xi) d \tau d \xi \\
& +\frac{1}{p_{1}^{\alpha} \Gamma(\alpha)} \frac{1}{p_{2}^{\beta} \Gamma(\beta)} \\
& \int_{0}^{x} \int_{0}^{x} e^{\frac{p_{1}-1}{p_{1}}(x-\tau)} e^{\frac{p_{2}-1}{p_{2}}(x-\xi)}(x-\tau)^{\alpha-1}(x-\xi)^{\beta-1} v_{2}(\tau) f(\tau) w_{2}(\xi) g(\xi) d \tau d \xi \\
& \geq \frac{1}{p_{1}^{\alpha} \Gamma(\alpha)} \frac{1}{p_{2}^{\beta} \Gamma(\beta)} \\
& \int_{0}^{x} \int_{0}^{x} e^{\frac{p_{1}-1}{p_{1}}(x-\tau)} e^{\frac{p_{2}-1}{p_{2}}(x-\xi)}(x-\tau)^{\alpha-1}(x-\xi)^{\beta-1} w_{1}(\xi) w_{2}(\xi) f^{2}(\tau) d \tau d \xi \\
& +\frac{1}{p_{1}^{\alpha} \Gamma(\alpha)} \frac{1}{p_{2}^{\beta} \Gamma(\beta)} \\
& \int_{0}^{x} \int_{0}^{x} e^{\frac{p_{1}-1}{p_{1}}(x-\tau)} e^{\frac{p_{2}-1}{p_{2}}(x-\xi)}(x-\tau)^{\alpha-1}(x-\xi)^{\beta-1} v_{1}(\tau) v_{2}(\tau) g(\xi) d \tau d \xi .
\end{aligned}
$$

If we re-write the above inequality with the help of the definition of operator, we get

$$
\begin{aligned}
& { }_{0}^{G P F} I^{\alpha, p_{1}}\left[v_{1} f\right](x)_{0}^{G P F} I^{\beta, p_{2}}\left[w_{1} g\right](x)+{ }_{0}^{G P F} I^{\alpha, p_{1}}\left[v_{2} f\right](x)_{0}^{G P F} I^{\beta, p_{2}}\left[w_{2} g\right](x) \\
\geq & { }_{0}^{G P F} I^{\alpha, p_{1}}\left[f^{2}\right](x)_{0}^{G P F} I^{\beta, p_{2}}\left[w_{1} w_{2}\right](x)+{ }_{0}^{G P F} I^{\alpha, p_{1}}\left[v_{1} v_{2}\right](x)_{0}^{G P F} I^{\beta, p_{2}}\left[g^{2}\right](x) .
\end{aligned}
$$

Applying the A.M-G.M inequality, we have

$$
\begin{aligned}
{ }_{0}^{G P F} I^{\alpha, p_{1}}\left[v_{1} f\right](x)_{0}^{G P F} I^{\beta, p_{2}}\left[w_{1} g\right](x)+{ }_{0}^{G P F} I^{\alpha, p_{1}}\left[v_{2} f\right](x)_{0}^{G P F} I^{\beta, p_{2}}\left[w_{2} g\right](x) \\
\geq 2 \sqrt{{ }_{0}^{G P F} I^{\alpha, p_{1}}\left[f^{2}\right](x)_{0}^{G P F} I^{\beta, p_{2}}\left[w_{1} w_{2}\right](x) \times{ }_{0}^{G P F} I^{\alpha, p_{1}}\left[v_{1} v_{2}\right](x)_{0}^{G P F} I^{\beta, p_{2}}\left[g^{2}\right](x) .}
\end{aligned}
$$

Which leads to the desired inequality in (2.7). The proof is completed. 
Corollary 2. If we set $v_{1}=M, v_{2}=M, w_{1}=n$ and $w_{2}=N$ in (2.7), then we have the following inequality;

${ }_{0}^{G P F} I^{\alpha, p_{1}}(x)_{0}^{G P F} I^{\beta, p_{2}}(x) \frac{\left({ }_{0}^{G P F} I^{\alpha, p_{1}} f^{2}\right)(x)\left({ }_{0}^{G P F} I^{\beta, p_{2}} g^{2}\right)(x)}{\left(\left({ }_{0}^{G P F} I^{\alpha, p_{1}} f\right)(x)\left({ }_{0}^{G P F} I^{\beta, p_{2}} g\right)(x)\right)^{2}} \leq \frac{1}{4}\left(\sqrt{\frac{m n}{M N}}+\sqrt{\frac{M N}{m n}}\right)^{2}$

Let $a=\frac{p_{1}-1}{p_{1}}$. The Taylor Series of $\exp (a(x-\tau))$ at the point $x$ is given by

$$
\begin{aligned}
& { }_{0}^{G P F} I^{\alpha, p_{1}}(x)=\frac{1}{p_{1}^{\alpha} \Gamma(\alpha)} \int_{0}^{x} e^{\frac{p_{1}-1}{p_{1}}(x-\tau)}(x-\tau)^{\alpha-1} d \tau \\
& =\frac{1}{p_{1} \Gamma(\alpha)} \int_{0}^{x} \sum_{k=0}^{\infty} \frac{(a(x-\tau))^{k_{1}}}{k_{1} !}(x-\tau)^{\alpha-1} d \tau \\
& =\frac{1}{p_{1}^{\alpha} \Gamma(\alpha)} \sum_{k=0}^{\infty} \frac{1}{k !} \frac{a^{k_{1}} x^{\alpha+k_{1}}}{\alpha+k_{1}} \\
& { }_{0}^{G P F} I^{\beta, p_{2}}(x)=\frac{1}{p_{2}^{\beta} \Gamma(\beta)} \sum_{k=0}^{\infty} \frac{b^{k_{2}} x^{\beta+k_{2}}}{k_{2} !\left(\beta+k_{2}\right)} \\
& \frac{1}{p_{1}^{\alpha} \Gamma(\alpha)} \frac{1}{p_{2}^{\beta} \Gamma(\beta)} \sum_{k_{1}=0}^{\infty} \frac{a^{k_{1}} x^{\alpha+k_{1}}}{\left(\alpha+k_{1}\right) k !} \sum_{k_{2}=0}^{\infty} \frac{b^{k_{2}} x^{\beta+k_{2}}}{\left(\beta+k_{2}\right) k_{2} !} \\
& \times \frac{\left({ }_{0}^{G P F} I^{\alpha, p_{1}} f^{2}\right)(x)\left({ }_{0}^{G P F} I^{\beta, p_{2}} g^{2}\right)(x)}{\left(\left({ }_{0}^{G P F} I^{\alpha, p_{1}} f\right)(x)\left({ }_{0}^{G P F} I^{\beta, p_{2}} g\right)(x)\right)^{2}} \\
& \leq \frac{1}{4}\left(\sqrt{\frac{m n}{M N}}+\sqrt{\frac{M N}{m n}}\right)^{2} .
\end{aligned}
$$

Lemma 3. Let $f$ and $g$ be two positive integrable function on $[0, \infty)$. Assume that there exist four positive integrable functions $v_{1}, v_{2}, w_{1}$ and $w_{2}$ satisfying condition (2.1) then the following inequality holds.

$$
{ }_{0}^{G P F} I^{\alpha, p_{1}}\left[f^{2}\right](x)_{0}^{G P F} I^{\beta, p_{2}}\left[g^{2}\right](x) \leq{ }_{0}^{G P F} I^{\alpha, p_{1}}\left[\frac{v_{2} f g}{w_{1}}\right](x)_{0}^{G P F} I^{\beta, p_{2}}\left[\frac{w_{2} f g}{v_{1}}\right](x) .
$$

where $\alpha \in(n, n+1], \beta \in(k, k+1], n, k=0,1,2,3, \ldots$.

Proof. Using the condition (2.1), we get

$$
f^{2}(\tau) \leq \frac{v_{2}(\tau)}{w_{1}(\tau)} f(\tau) g(\tau)
$$


Multiplying both sides of (2.11) by $\frac{1}{p_{1}^{\alpha} \Gamma(\alpha)} e^{\frac{p_{1}-1}{p_{1}}(x-\tau)}(x-\tau)^{\alpha-1}$ and integrating the resulting inequality with respect to $\tau$ over $(0, x)$, we get

$$
\begin{gathered}
\frac{1}{p_{1}^{\alpha} \Gamma(\alpha)} \int_{0}^{x} e^{\frac{p_{1}-1}{p_{1}}(x-\tau)}(x-\tau)^{\alpha-1} f^{2}(\tau) d \tau \\
\leq \frac{1}{p_{1}^{\alpha} \Gamma(\alpha)} \int_{0}^{x} e^{\frac{p_{1}-1}{p_{1}}(x-\tau)}(x-\tau)^{\alpha-1} \frac{v_{2} \tau}{w_{1}(\tau)} f(\tau) g(\tau) d \tau \\
{ }_{0}^{G P F} I^{\alpha, p_{1}}\left[f^{2}\right](x) \leq{ }_{0}^{G P F} I^{\alpha, p}\left[\frac{v_{2} f g}{w_{1}}\right](x) .
\end{gathered}
$$

Similarly, we can write

$$
g^{2}(\xi) \leq \frac{w_{2}(\xi)}{v_{1}(\xi)} f(\xi) g(\xi)
$$

By a similar argument, we have

$$
\begin{gathered}
\frac{1}{p_{2}^{\beta} \Gamma(\beta)} \int_{0}^{x} e^{\frac{p_{2}-1}{p_{2}}(x-\xi)}(x-\xi)^{\beta-1} g^{2}(\xi) d \xi \\
\leq \frac{1}{p_{2}^{\beta} \Gamma(\beta)} \int_{0}^{x} e^{\frac{p_{2}-1}{p_{2}}(x-\xi)}(x-\xi)^{\beta-1} \frac{w_{2}(\xi)}{v_{1}(\xi)} f(\xi) g(\xi) d \xi .
\end{gathered}
$$

Which implies

$$
{ }_{0}^{G P F} I^{\beta, p_{2}}\left[g^{2}\right](x) \leq{ }_{0}^{G P F} I^{\beta, p_{2}}\left[\frac{w_{2} f g}{v_{1}}\right](x) .
$$

Multiplying (2.12) and (2.13), we get then (2.10). Then the desired inequality is obtained such that

$$
{ }_{0}^{G P F} I^{\alpha, p_{1}}\left[f^{2}\right](x)_{0}^{G P F} I^{\beta, p_{2}}\left[g^{2}\right](x) \leq{ }_{0}^{G P F} I^{\alpha, p_{1}}\left[\frac{v_{2} f g}{w_{1}}\right](x)_{0}^{G P F} I^{\beta, p_{2}}\left[\frac{w_{2} f g}{v_{1}}\right](x) .
$$

Corollary 3. If we choose $v_{1}=m, v_{2}=M, w_{1}=n$ and $w_{2}=N$ in (2.10), then we have the following inequality;

$$
\frac{\left(\begin{array}{l}
G P F \\
0
\end{array} I^{\alpha, p_{1}} f^{2}\right)(x)\left(\begin{array}{l}
G P F \\
0
\end{array} I^{\alpha, p_{1}} g^{2}\right)(x)}{\left({ }_{0}^{G P F} I^{\alpha, p_{1}} f g\right)(x)\left({ }_{0}^{G P F} I^{\alpha, p_{1}} f g\right)(x)} \leq \frac{M N}{m n} .
$$

Theorem 2. Let $f$ and $g$ be two positive integrable function on $[0, \infty)$. Assume that there exist four positive integrable functions $v_{1}, v_{2}, w_{1}$ and $w_{2}$ satisfying condition 
(2.1) then the following inequality holds:

$$
\begin{gathered}
\left(\frac{1}{p_{1}^{\alpha} \Gamma(\alpha)} \sum_{k_{1}=0}^{\infty} \frac{a^{k_{1}}}{k_{1} !} \frac{x^{\alpha+k_{1}}}{\alpha+k_{1}}\right)\left({ }_{0}^{G P F} I^{\beta, p_{2}} f g\right)(x) \\
+\left(\frac{1}{p_{2}^{\beta} \Gamma(\beta)} \sum_{k_{2}=0}^{\infty} \frac{b^{k_{2}}}{k_{2} !} \frac{x^{\beta+k_{2}}}{\beta+k_{2}}\right)\left({ }_{{ }_{0}^{G P F}} I^{\alpha, p_{1}} f g\right)(x) \\
-\left({ }_{0}^{G P F} I^{\alpha, p_{1}} f\right)(x)\left({ }_{0}^{G P F} I^{\beta, p_{2}} g\right)(x)-\left({ }_{0}^{G P F} I^{\beta, p_{2}} f\right)(x)\left({ }_{0}^{G P F} I^{\alpha, p_{1}} g\right)(x) \\
\leq\left|A_{1}\left(f, v_{1}, v_{2}\right)(x)+A_{2}\left(f, v_{1}, v_{2}\right)(x)\right|^{\frac{1}{2}} \times\left|A_{1}\left(g, w_{1}, w_{2}\right)(x)+A_{2}\left(g, w_{1}, w_{2}\right)(x)\right|^{\frac{1}{2}}, \\
\text { for } \alpha \in(n, n+1], \beta \in(k, k+1], n, k=0,1,2,3, \ldots, \text { where }
\end{gathered}
$$

$$
\begin{gathered}
A_{1}(u, v, w)(x)=\left(\frac{1}{p_{2}^{\beta} \Gamma(\beta)} \sum_{k_{2}=0}^{\infty} \frac{b^{k_{2}}}{k_{2} !} \frac{x^{\beta+k_{2}}}{\beta+k_{2}}\right) \\
\times \frac{\left({ }_{0}^{G P F} I^{\alpha, p_{1}}[(v+w) u](x)\right)^{2}}{4_{0}^{G P F} I^{\alpha, p_{1}[v w](x)}}-\left({ }_{0}^{G P F} I^{\alpha, p_{1}} u\right)(x)\left({ }_{0}^{G P F} I^{\beta, p_{2}} u\right)(x)
\end{gathered}
$$

and

$$
\begin{gathered}
A_{2}(u, v, w)(x)=\left(\frac{1}{p_{1}^{\alpha} \Gamma(\alpha)} \sum_{k_{1}=0}^{\infty} \frac{a^{k_{1}}}{k_{1} !} \frac{x^{\alpha+k_{1}}}{\alpha+k_{1}}\right) \\
\times \frac{\left({ }_{0}^{G P F} I^{\beta, p_{2}}[(v+w) u](x)\right)^{2}}{4_{0}^{G P F} I^{\beta, p_{2}}[v w](x)}-\left({ }_{0}^{G P F} I^{\alpha, p_{1}} u\right)(x)\left({ }_{0}^{G P F} I^{\beta, p_{2}} u\right)(x) .
\end{gathered}
$$

Proof. Let $f$ and $g$ be two positive integrable functions on $[0, \infty)$ for $\tau, \xi \in(0, x)$ with $x>0$, we define $H(\tau, \xi)$ as

$$
H(\tau, \xi)=(f(\tau)-f(\xi))(g(\tau)-g(\xi))
$$

Namely

$$
H(\tau, \xi)=f(\tau) g(\tau)+f(\xi) g(\xi)-f(\tau) g(\xi)-f(\xi) g(\tau) .
$$

Multiplying both sides of (2.14) by

$$
\frac{1}{p_{1}^{\alpha} \Gamma(\alpha)} \frac{1}{p_{2}^{\beta} \Gamma(\beta)}(x-\tau)^{\alpha-1}(x-\xi)^{\beta-1} e^{\frac{p_{1}-1}{p_{1}}(x-\tau)} e^{\frac{p_{2}-1}{p_{2}}(x-\xi)} .
$$

Then by integrating the resulting inequality with respect to $\tau$ and $\xi$ over $(0, x)^{2}$, we get

$$
\frac{1}{p_{1}^{\alpha} \Gamma(\alpha)} \frac{1}{p_{2}^{\beta} \Gamma(\beta)} \int_{0}^{x} \int_{0}^{x} e^{\frac{p_{1}-1}{p_{1}}(x-\tau)} e^{\frac{p_{2}-1}{p_{2}}(x-\xi)}(x-\tau)^{\alpha-1}(x-\xi)^{\beta-1} H(\tau, \xi) d \tau d \xi
$$


726 SAAD IHSAN BUTT, AHMET OCAK AKDEMIR, ALPER EKINCI, AND MUHAMMAD NADEEM

$$
\begin{gathered}
=\frac{1}{p_{1}^{\alpha} \Gamma(\alpha)} \frac{1}{p_{2}^{\beta} \Gamma(\beta)} \int_{0}^{x} \int_{0}^{x} e^{\frac{p_{1}-1}{p_{1}}(x-\tau)} e^{\frac{p_{2}-1}{p_{2}}(x-\xi)}(x-\tau)^{\alpha-1}(x-\xi)^{\beta-1} f(\tau) g(\tau) d \tau d \xi \\
+\frac{1}{p_{1}^{\alpha} \Gamma(\alpha)} \frac{1}{p_{2}^{\beta} \Gamma(\beta)} \int_{0}^{x} \int_{0}^{x} e^{\frac{p_{1}-1}{p_{1}}(x-\tau)} e^{\frac{p_{2}-1}{p_{2}}(x-\xi)}(x-\tau)^{\alpha-1}(x-\xi)^{\beta-1} f(\xi) g(\xi) d \tau d \xi \\
-\frac{1}{p_{1}^{\alpha} \Gamma(\alpha)} \frac{1}{p_{2}^{\beta} \Gamma(\beta)} \int_{0}^{x} \int_{0}^{x} e^{\frac{p_{1}-1}{p_{1}}(x-\tau)} e^{\frac{p_{2}-1}{p_{2}}(x-\xi)}(x-\tau)^{\alpha-1}(x-\xi)^{\beta-1} f(\tau) g(\xi) d \tau d \xi \\
-\frac{1}{p_{1}^{\alpha} \Gamma(\alpha)} \frac{1}{p_{2}^{\beta} \Gamma(\beta)} \int_{0}^{x} \int_{0}^{x} e^{\frac{p_{1}-1}{p_{1}}(x-\tau)} e^{\frac{p_{2}-1}{p_{2}}(x-\xi)}(x-\tau)^{\alpha-1}(x-\xi)^{\beta-1} f(\xi) g(\tau) d \tau d \xi \\
=\left(\frac{1}{p_{2}^{\beta} \Gamma(\beta)} \sum_{k_{2}=0}^{\infty} \frac{b^{k_{2}}}{k_{2} !} \frac{x^{\beta+k_{2}}}{\beta+k_{2}}\right)\left({ }_{G}^{G P F} I^{\alpha, p_{1}} f g\right)(x) \\
+\left(\frac{1}{p_{1}^{\alpha} \Gamma(\alpha)} \sum_{k_{1}=0}^{\infty} \frac{a^{k_{1}}}{k_{1} !} \frac{x^{\alpha+k_{1}}}{\alpha+k_{1}}\right)\left(\begin{array}{l}
G P F \\
0
\end{array} I^{\beta, p_{2}} f g\right)(x) \\
-\left(\begin{array}{l}
G P F \\
0
\end{array} I^{\alpha, p_{1}} f\right)(x)\left(\begin{array}{l}
G P F \\
0
\end{array} I^{\beta, p_{2}} g\right)(x)-\left(\begin{array}{l}
G P F \\
0
\end{array} I^{\beta, p_{2}} f\right)(x)\left(\begin{array}{l}
G P F \\
0
\end{array} I^{\alpha, p_{1}} g\right)(x) .
\end{gathered}
$$


Applying the Cauchy-Schwarz inequality, we can write

$$
\begin{aligned}
& \mid \frac{1}{p_{1}^{\alpha} \Gamma(\alpha)} \frac{1}{p_{2}^{\beta} \Gamma(\beta)} \int_{0}^{x} \int_{0}^{x} e^{\frac{p_{1}-1}{p_{1}}(x-\tau)} e^{\frac{p_{2}-1}{p_{2}}(x-\xi)}(x-\tau)^{\alpha-1}(x-\xi)^{\beta-1} H(\tau, \xi) d \tau d \xi \mid \\
& \leq {\left[\frac{1}{p_{1}^{\alpha} \Gamma(\alpha)} \frac{1}{p_{2}^{\beta} \Gamma(\beta)} \int_{0}^{x} \int_{0}^{x} e^{\frac{p_{1}-1}{p_{1}}(x-\tau)} e^{\frac{p_{2}-1}{p_{2}}(x-\xi)}(x-\tau)^{\alpha-1}(x-\xi)^{\beta-1} f^{2}(\tau) d \tau d \xi\right.} \\
&+\frac{1}{p_{1}^{\alpha} \Gamma(\alpha)} \frac{1}{p_{2}^{\beta} \Gamma(\beta)} \int_{0}^{x} \int_{0}^{x} e^{\frac{p_{1}-1}{p_{1}}(x-\tau)} e^{\frac{p_{2}-1}{p_{2}}(x-\xi)}(x-\tau)^{\alpha-1}(x-\xi)^{\beta-1} f^{2}(\xi) d \tau d \xi \\
&\left.-2 \frac{1}{p_{1}^{\alpha} \Gamma(\alpha)} \frac{1}{p_{2}^{\beta} \Gamma(\beta)} \int_{0}^{x} \int_{0}^{x} e^{\frac{p_{1}-1}{p_{1}}(x-\tau)} e^{\frac{p_{2}-1}{p_{2}}(x-\xi)}(x-\tau)^{\alpha-1}(x-\xi)^{\beta-1} f(\tau) f(\xi) d \tau d \xi\right]^{\frac{1}{2}} \\
& \quad {\left[\frac{1}{p_{1}^{\alpha} \Gamma(\alpha)} \frac{1}{p_{2}^{\beta} \Gamma(\beta)} \int_{0}^{x} \int_{0}^{x} e^{\frac{p_{1}-1}{p_{1}}(x-\tau)} e^{\frac{p_{2}-1}{p_{2}}(x-\xi)}(x-\tau)^{\alpha-1}(x-\xi)^{\beta-1} g^{2}(\tau) d \tau d \xi\right.} \\
& \quad+\frac{1}{p_{1}^{\alpha} \Gamma(\alpha)} \frac{1}{p_{2}^{\beta} \Gamma(\beta)} \int_{0}^{x} \int_{0}^{x} e^{\frac{p_{1}-1}{p_{1}}(x-\tau)} e^{\frac{p_{2}-1}{p_{2}}(x-\xi)}(x-\tau)^{\alpha-1}(x-\xi)^{\beta-1} g^{2}(\xi) d \tau d \xi \\
&\left.-2 \frac{1}{p_{1}^{\alpha} \Gamma(\alpha)} \frac{1}{p_{2}^{\beta} \Gamma(\beta)} \int_{0}^{x} \int_{0}^{x} e^{\frac{p_{1}-1}{p_{1}}(x-\tau)} e^{\frac{p_{2}-1}{p_{2}}(x-\xi)}(x-\tau)^{\alpha-1}(x-\xi)^{\beta-1} g(\tau) g(\xi) d \tau d \xi\right]^{\frac{1}{2}} .
\end{aligned}
$$


As a consequence

$$
\begin{aligned}
& \left|\frac{1}{p_{1}^{\alpha} \Gamma(\alpha)} \frac{1}{p_{2}^{\beta} \Gamma(\beta)} \int_{0}^{x} \int_{0}^{x} e^{\frac{p_{1}-1}{p_{1}}(x-\tau)} e^{\frac{p_{2}-1}{p_{2}}(x-\xi)}(x-\tau)^{\alpha-1}(x-\xi)^{\beta-1} H(\tau, \xi) d \tau d \xi\right| \\
& \leq\left[\left(\frac{1}{p_{2}^{\beta} \Gamma(\beta)} \sum_{k_{2}=0}^{\infty} \frac{b^{k_{2}}}{k_{2} !} \frac{x^{\beta+k_{2}}}{\beta+k_{2}}\right)\left({ }_{0}^{G P F} I^{\alpha, p_{1}} f^{2}\right)(x)\right. \\
& +\left(\frac{1}{p_{1}^{\alpha} \Gamma(\alpha)} \sum_{k_{1}=0}^{\infty} \frac{a^{k_{1}}}{k_{1} !} \frac{x^{\alpha+k_{1}}}{\alpha+k_{1}}\right)\left({ }_{0}^{G P F} I^{\beta, p_{2}} f^{2}\right)(x) \\
& \left.-2\left({ }_{0}^{G P F} I^{\beta, p_{2}} f\right)(x)\left({ }_{0}^{G P F} I^{\alpha, p_{1}} f\right)(x)\right]^{\frac{1}{2}} \\
& +\left(\frac{1}{p_{2}^{\beta} \Gamma(\beta)} \sum_{k_{2}=0}^{\infty} \frac{b^{k_{2}}}{k_{2} !} \frac{x^{\beta+k_{2}}}{\beta+k_{2}}\right)\left({ }_{0}^{G P F} I^{\alpha, p_{1}} g^{2}\right)(x) \\
& +\left(\frac{1}{p_{1}^{\alpha} \Gamma(\alpha)} \sum_{k_{1}=0}^{\infty} \frac{a^{k_{1}}}{k_{1} !} \frac{x^{\alpha+k_{1}}}{\alpha+k_{1}}\right)\left({ }_{G}^{G P F} I^{\beta, p_{2}} g^{2}\right)(x) \\
& \left.-2\left({ }_{0}^{G P F} I^{\beta, p_{2}} g\right)(x)\left({ }_{0}^{G P F} I^{\alpha, p_{1}} g\right)(x)\right]^{\frac{1}{2}} .
\end{aligned}
$$

Applying Lemma 1 with $w_{1}(\tau)=w_{2}(\tau)=g(\tau)=1$, we get

$$
\begin{aligned}
& \left(\frac{1}{p_{2}^{\beta} \Gamma(\beta)} \sum_{k_{2}=0}^{\infty} \frac{b^{k_{2}}}{k_{2} !} \frac{x^{\beta+k_{2}}}{\beta+k_{2}}\right)\left({ }_{0}^{G P F} I^{\alpha, p_{1}} f^{2}\right)(x) \\
& \leq\left(\frac{1}{p_{2}^{\beta} \Gamma(\beta)} \sum_{k_{2}=0}^{\infty} \frac{b^{k_{2}}}{k_{2} !} \frac{x^{\beta+k_{2}}}{\beta+k_{2}}\right) \frac{\left({ }_{0}^{G P F} I^{\alpha, p_{1}}\left[\left(v_{1}+v_{2}\right) f\right](x)\right)^{2}}{4_{0}^{G P F} I^{\alpha, p_{1}}\left[v_{1} v_{2}\right](x)} .
\end{aligned}
$$

This implies that

$$
\begin{aligned}
& \left(\frac{1}{p_{2}^{\beta} \Gamma(\beta)} \sum_{k_{2}=0}^{\infty} \frac{b^{k_{2}}}{k_{2} !} \frac{x^{\beta+k_{2}}}{\beta+k_{2}}\right)\left({ }_{0}^{G P F} I^{\alpha, p_{1}} f^{2}\right)(x)-\left({ }_{0}^{G P F} I^{\alpha, p_{1}} f\right)(x)\left({ }_{0}^{G P F} I^{\beta, p_{2}} f\right)(x) \\
& \leq\left(\frac{1}{p_{2}^{\beta} \Gamma(\beta)} \sum_{k_{2}=0}^{\infty} \frac{b^{k_{2}}}{k_{2} !} \frac{x^{\beta+k_{2}}}{\beta+k_{2}}\right) \frac{\left({ }_{0}^{G P F} I^{\alpha, p_{1}}\left[\left(v_{1}+v_{2}\right) f\right](x)\right)^{2}}{4_{0}^{G P F} I^{\alpha, p_{1}}\left[v_{1} v_{2}\right](x)} \\
& -\left({ }_{0}^{G P F} I^{\alpha, p_{1}} f\right)(x)\left({ }_{0}^{G P F} I^{\beta, p_{2}} f\right)(x)=A_{1}\left(f, v_{1}, v_{2}\right)
\end{aligned}
$$


and

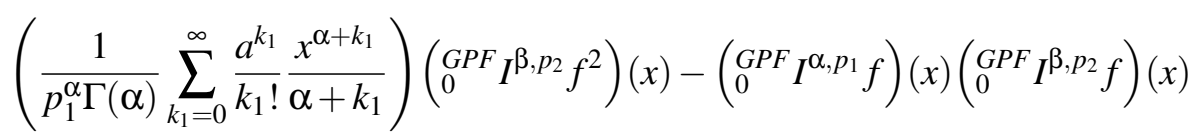

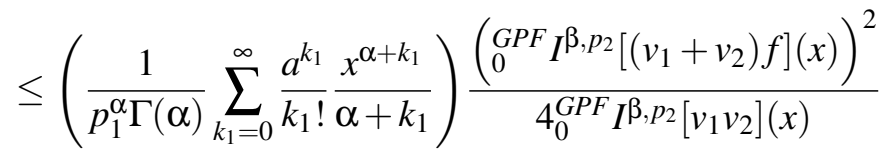

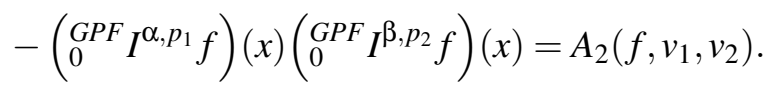

Similarly, applying Lemma 1 with $v_{1}(\tau)=v_{2}(\tau)=f(\tau)=1$, we have

$$
\begin{gathered}
\left(\frac{1}{p_{2}^{\beta} \Gamma(\beta)} \sum_{k_{2}=0}^{\infty} \frac{b^{k_{2}}}{k_{2} !} \frac{x^{\beta+k_{2}}}{\beta+k_{2}}\right){ }_{0}^{G P F} I^{\alpha, p_{1}}\left[g^{2}\right](x) \\
-\left({ }_{0}^{G P F} I^{\alpha, p_{1}} g\right)(x)\left(\begin{array}{l}
G P F \\
0
\end{array} I^{\beta, p_{2}} g\right)(x) \leq A_{1}\left(g, w_{1}, w_{2}\right)
\end{gathered}
$$

and

$$
\begin{aligned}
& \left(\frac{1}{p_{1}^{\alpha} \Gamma(\alpha)} \sum_{k_{1}=0}^{\infty} \frac{a^{k_{1}}}{k_{1} !} \frac{x^{\alpha+k_{1}}}{\alpha+k_{1}}\right)_{0}^{G P F} I^{\beta, p_{2}}\left[g^{2}\right](x) \\
& -\left(\begin{array}{l}
G P F \\
0
\end{array} I^{\alpha, p_{1}} g\right)(x)\left(\begin{array}{l}
G P F \\
0
\end{array} I^{\beta, p_{2}} g\right)(x) \leq A_{2}\left(g, w_{1}, w_{2}\right) \text {. }
\end{aligned}
$$

Using (2.15)-(2.18), we conclude the result.

Theorem 3. Let $f$ and $g$ be two positive integrable function on $[0, \infty)$. Assume that there exist four positive integrable functions $v_{1}, v_{2}, w_{1}$ and $w_{2}$ satisfying condition (2.1) then the following inequality holds:

$$
\begin{gathered}
\left|\left(\frac{1}{p_{1}^{\alpha} \Gamma(\alpha)} \sum_{k_{1}=0}^{\infty} \frac{a^{k_{1}}}{k_{1} !} \frac{x^{\alpha+k_{1}}}{\alpha+k_{1}}\right){ }_{0}^{G P F} I^{\alpha, p_{1}}[f g](x)-\left({ }_{0}^{G P F} I^{\alpha, p_{1}} f\right)(x)\left({ }_{0}^{G P F} I^{\alpha, p_{1}} g\right)(x)\right| \\
\leq\left|A\left(f, v_{1}, v_{2}\right)(x) A\left(g, w_{1}, w_{2}\right)(x)\right|^{\frac{1}{2}}
\end{gathered}
$$

for $\alpha \in(n, n+1], \beta \in(k, k+1], n, k=0,1,2,3, \ldots$, where

$$
A(u, v, w)(x)=\left(\frac{1}{p_{1}^{\alpha} \Gamma(\alpha)} \sum_{k_{1}=0}^{\infty} \frac{a^{k_{1}}}{k_{1} !} \frac{x^{\alpha+k_{1}}}{\alpha+k_{1}}\right)
$$




$$
\times \frac{\left({ }_{0}^{G P F} I^{\alpha, p_{1}}[(v+w) u](x)\right)^{2}}{4_{0}^{G P F} I^{\alpha, p_{1}}[v w](x)}-\left(\left({ }_{0}^{G P F} I^{\alpha, p_{1}} u\right)(x)\right)^{2} .
$$

Proof. Setting $\alpha=\theta$ in Theorem 2, we obtain (2.19).

Corollary 4. Assume that all the assumptions of Theorem 3 satify, then we have the following inequality;

$$
\begin{aligned}
& \left|\left(\frac{1}{p_{1}^{\alpha} \Gamma(\alpha)} \sum_{k_{1}=0}^{\infty} \frac{a^{k_{1}}}{k_{1} !} \frac{x^{\alpha+k_{1}}}{\alpha+k_{1}}\right){ }_{0}^{G P F} I^{\alpha, p_{1}}[f g](x)-\left({ }_{0}^{G P F} I^{\alpha, p_{1}} f\right)(x)\left({ }_{0}^{G P F} I^{\alpha, p_{1}} g\right)(x)\right| \\
\leq & \left(\frac{1}{p_{1}^{\alpha} \Gamma(\alpha)} \sum_{k_{1}=0}^{\infty} \frac{a^{k_{1}}}{k_{1} !} \frac{x^{\alpha+k_{1}}}{\alpha+k_{1}}\right) \frac{(M-m)(N-n)}{4 \sqrt{M m N n}} \times\left({ }_{0}^{G P F} I^{\alpha, p_{1}} f\right)(x)\left({ }_{0}^{G P F} I^{\alpha, p_{1}} g\right)(x) .
\end{aligned}
$$

Proof. If we set $v_{1}=m, v_{2}=M, w_{1}=n$ and $w_{2}=N$ in (2.19), then the proof is completed. We omit the details.

\section{CONFLiCT OF INTEREST}

All authors declare no conflicts of interest in this paper.

\section{ACKNOWLEDGEMENT}

The research of the first author has been fully supported by H.E.C. Pakistan under NRPU project 7906.

\section{REFERENCES}

[1] H. Budak and P. Agarwal, "New generalized Midpoint type inequalities for fractional integral," Miskolc Math. Notes, vol. 20, pp. 781-793, 2019, doi: 10.18514/MMN.2019.2525.

[2] P. L. Chebyshev, "Sur les expressions approximatives des integrales definies par les autres prises entre les memes limites," Proc. Math. Soc. Charkov, vol. 2, pp. 93-98, 1882.

[3] Z. Dahmani, O. Mechouar, and S. Brahami, "Certain inequalities related to the Chebyshev functional involving a type Riemann-Liouville operator," Bull. Math. Anal. Appl., vol. 3, pp. 38-44, 2011.

[4] M. A. Dokuyucu, "A fractional order alcoholism model via Caputo-Fabrizio derivative," AIMS Mathematics, vol. 5, no. 2, pp. 781-797, 2020, doi: 10.3934/math.2020053.

[5] M. A. Dokuyucu, E. Celik, H. Bulut et al., "Cancer treatment model with the CaputoFabrizio fractional derivative,” The European Physical Journal Plus, vol. 133, p. 92, 2018, doi: 10.1140/epjp/i2018-11950-y.

[6] M. A. Dokuyucu and H. Dutta, "A fractional order model for Ebola Virus with the new Caputo fractional derivative without singular kernel," Chaos, Solitons and Fractals, vol. 134, May 2020, doi: 10.1016/j.chaos.2020.109717.

[7] S. S. Dragomir and N. T. Diamond, "Integral inequalities of Gruss type via Polya-Szego and Shisha-Mond results," East Asian Math J., vol. 19, pp. 27-39, 2003.

[8] A. Ekinci and M. E. Ozdemir, "Some New Integral Inequalities Via Riemann Liouville Integral Operators," Applied and Computational Mathematics, vol. 3, pp. 288-295, 2019. 
[9] G. Farid, "Existence of an integral operator and its consequences in fractional and conformable integrals," Open Journal of Mathematical Sciences, vol. 3, pp. 210-216, 2019, doi: 10.30538/oms2019.0064.

[10] S. Gala and M. Ragusa, "Logarithmically improved regularity criterion for the Boussinesq equations in Besov spaces with negative indices," Applicable Analysis, vol. 95, pp. 1271-1279, 2016, doi: 10.1080/00036811.2015.1061122.

[11] F. Jarad, T. Abdeljawad, and J. Alzabut, "Generalized fractional derivatives generated by a class of local proportional derivatives," Eur. Phys. J. Spec. Top., vol. 226, p. 34573471, 2017, doi: 10.1140/epjst/e2018-00021-7.

[12] A. A. Kilbas, Theory and applications of fractional differential equations. Amsterdam Boston: Elsevier, 2006.

[13] D. Nie, S. Rashid, A. O. Akdemir, D. Baleanu, and J.-B. Liu, "On Some New Weighted Inequalities for Differentiable Exponentially Convex and Exponentially Quasi-Convex Functions with Applications," Mathematics, vol. 7, no. 8, p. 727, 2019, doi: 10.3390/math7080727.

[14] S. K. Ntouyas, P. Agarwal, and J. Tariboon, "On Polya-Szegö and Chebyshev type inequalities involving the Riemann-Liouville fractional integral operators," J. Math. Inequal, vol. 10, pp. 491504, 2016, doi: 10.7153/jmi-10-38.

[15] M. E. Ozdemir, E. Set, O. A. Akdemir et al., "Some new Chebyshev type inequalities for functions whose derivatives belongs to Lp spaces," Afr. Mat., vol. 26, pp. 1609-1619, 2015, doi: 10.1007/s13370-014-0312-5.

[16] I. Podlubny, Fractional differential equations: an introduction to fractional derivatives, fractional differential equations, to methods of their solution and some of their applications. San Diego: Academic Press, 1999.

[17] S. Polidoro and M. Ragusa, "Harnack inequality for hypoelliptic ultraparabolic equations with a singular lower order term," Revista Matematica Iberoamericana, vol. 24, pp. 1011-1046, 2008.

[18] G. Polya, Aufgaben und Lehrsatze aus der Analysis. Berlin, Heidelberg: Springer Berlin Heidelberg Imprint Springer, 1925.

[19] M. Z. Sarikaya and N. Alp, "On Hermite-Hadamard-Fejer type integral inequalities for generalized convex functions via local fractional integrals," Open Journal of Mathematical Sciences, vol. 3, pp. 273-284, 2019, doi: 10.30538/oms2019.0070.

[20] M. Z. Sarikaya, E. Set, H. Yaldız, and N. Basak, "Hermite-Hadamard's inequalities for fractional integrals and related fractional inequalities," Mathematical and Computer Modelling, vol. 57, pp. 2403-2407, 2013, doi: 10.1016/j.mcm.2011.12.048.

[21] E. Set, A. O. Akdemir, and I. Mumcu, "Chebyshev type inequalities for conformable fractional integrals," Miskolc Mathematical Notes, vol. 20, pp. 1227-1236, 2019, doi: 10.18514/MMN.2019.2766.

Authors' addresses

Saad Ihsan Butt

COMSATS University Islamabad, Lahore Campus, Pakistan

E-mail address: saadihsanbuttegmail.com

\section{Ahmet Ocak Akdemir}

Department of Mathematics, Faculty of Science and Letters, Ağri Ibrahim Cecen University, Ağri, Turkey

E-mail address: aocakakdemiregmail.com 
732 SAAD IHSAN BUTT, AHMET OCAK AKDEMIR, ALPER EKINCI, AND MUHAMMAD NADEEM

Alper Ekinci

Department of Foreign Trade, Bandirma Vocational High School, Bandirma Onyedi Eylul University, Balikesir, Turkey

E-mail address: alperekinci@hotmail.com

\section{Muhammad Nadeem}

COMSATS University Islamabad, Lahore Campus, Pakistan

E-mail address: muhammadnadeem98847@gmail.com 